Aus dem pathologisch-anatomischen Institut in Wien. Vorstand: Prof. Weichsel ba um.

\title{
Über die Chromreaktion der Glandula coccygea und die Beziehungen dieser Drüse zum Nervus sympathicus.
}

\author{
Von
}

Privatdoz. Dr. Oskar Stoerk.

Mit zwei Textfiguren.

Dr. Thomson Walker beschäftigte sich 1897-1898 als Hospitant des Wiener pathologisch-anatomischen Instituts unter meiner Anleitung mit der normalen Anatomie und Histologie der Steissdrüse. Das zwei Jahre nach Walkers Abgange aus dem Institute in London fertiggestellte Manuskript kam infolge von Verzögerung durch äussere Umstände erst 1902 zur Übersetzung aus dem Englischen und schliesslich 1904 zur Drucklegung. ${ }^{2}$ ) Dem Originalmanuskript wurde (mit Ausnahme der Anmerkung über die Langerh a n sschen Zellhaufen S. 153) nichts Wesentliches hinzugefügt.

Die Angabe dieser Daten sei mit Rücksicht auf das Folgende entschuldigt. Im "Anatomischen Anzeiger" (25. Bd., S. 209-216, 27. Juli 1904) veröffentlichte vämlich $\mathrm{A}$. Schaper ${ }_{n}$ Einige Bemerkungen über das Wesen and die morphologische Stellung der Glandula coccygea (Glomus coccygeum)," deren Inhalt eine Kritik der. Walkerschen Arbeit bildet - in dem Sinne, dass deren histologische Befunde zwar "manches Neue bringen, ${ }^{\text {" dass aber }}$ das Kapitel über das Wesen der Drüse in einigen Punkten der Ergänzung bedürfe. Die morphologische Übereinstimmung, welche Walker zwischen der Glandula cocçgea und anderen "Drüsen ohne Ausführungsgang ${ }^{“}$ zu konstatieren suchte, könne ausschliesslich für eine derselben, die Carotica, Geltung haben; „eine eingehendere Benutzung der spezielleren Literatur über diesen Gegenstand" würde diesbezüglich Walker „auf etwas sicherere Wege geführt haben." Diesc speziellere Literatur der Carotica ist nach Schaper: Schaper (1892)²), Stilling (Rec. de l'univ. Lausanne 1892), K ohn $\left.(1900)^{3}\right)$. Die beiden letzteren Autoren baben in der Carotica chromaffine Zellen nachweisen können, Koln habe auch die histogenetische Beziehung der Carotica zum Sympathicus dargelegt, das gleiche Ja c obs s o n (1899) ) für die Coccygea, Walker jedoch die Prüfung der Chromreaktion

1) „Über die menschliche Steissdrüse." Archiv f. mikroskop. Anatomie. Bd. 64 (1901), S. 121.

2) Archir f. mikroskop. Anatomie, Bd. 40, S. 287.

3) Dasselbe Archiv, Bd. 56, S. 263.

4) Dasselbe Archiv, Bd. 53, S. 78. 
für die Coccygea unterlassen, und darum sei es noch nicht möglich, $n^{\text {ohne }}$ jedes Bedenken die Steissdrüse als eine Schwesterdrüse der Carotisdrüse zu bezeichnen und mit ihr in die Gruppe der Paraganglien einzuordnen."

In einem „P. S." der Mitteilung Schapers beisst es dann, er habe ,erst nach Drucklegung des Vorstehenden . . . . . . bei nochmaliger Einsicht der Walker schen Arbeit" die Anmerkung Walkers (auf der ersten Seite) gelesen, nämlich: „Aus äusseren Gründen hat sich die Drucklegung des Manuskriptes dieser Arbeit um mehr als zwei Jahre verzögert. Ich wäre seinerzeit nicht darauf verfallen, mich mit der Frage der Chromafinität der Coccygea zu beschäftigen, ich behalte mir vor, auf diese nunmehr aktuelle Frage später einmal einzugehen." Wenn auch $\mathrm{Schaper}$ bezüglich des ${ }_{\text {"seinerzeit }}$ " nicht wissen konnte, dass "mehr als zwei Jahre" tatsächlich ciner Verzögerung von sieben Jahren entsprochen hatte, bätte er doch auf die eben zitierte Bemerkung Walkers hin (Schapers Veröffentlichung erfolgte etwa ein halbes Jahr nach dem Erscheinen der Arbeit Walkers) demselben noch Frist gewähren können, das Versprechen bezüglich der Chromaffinitätsprüfung einzulösen. Damit wäre Schapers Mitteilung eigentlich gegenstandslos geworden; wenn er Walkers Arbeit ergänzen zu können glaubte, so wäre vielleicht zu erwarten gewesen, dass er selbst etwas Positives über die Chromaffinität, der Coccygea auszusagen gewusst hätte und damit hätte er sich auch die Aufstellung der (vor ihm schon durch Kohn formulierten) Hypothese erspart, die wohl so ziemlich das einzig Wesentliche des Inhaltes seiner Mitteilung darstellt: „Heute schon scheint es mir auf Grund der bisher bereits aufgedeckten Tatsachen über die Entwicklung und Struktur der Steissdrüse kaum noch zweifelhaft, dass die typischen Zellen derselben sich als chromaffine Elemente entpuppen werden." Wir können aus dem folgenden vorwegnehmen, dass sich diese Voraussage nicht erfüllt hat. Hiermit sei der Hauptsache nach die Erwiderung an $\mathrm{Schaper}$ beendet. Im folgenden soll über die Ergebnisse der im Titel gekennzeichneten Untersuchungen berichtet werden, welche im Einverständnis mit Walker aufgenommen wurden, nachdem Walker selbst (derzeit Vorstand eines chirurgischen Spitals in London) nicht in der Lage war, sich mit wünschenswerter Beschleunigung dem Thema zu widmen.

Es erscheint angezeigt, einleitend in aller Kürze die Entwicklung der Kenntnis der Chromreaktion zu skizzieren. (A usführl. diesbezügl. Ko hn, das chromaftine Tewebe, Ergebn. der Anat. u. Entw., 1902, S. 25t.) Seit ihrer Entdeckung durch Heule an den Markzellen der Nebenniere (1865) hat sie sich, wie man nunmehr wohl schon sagen darf, geradezu als eine spezifische Reaktion für eine bestimmte Gewebsart erwiesen, welche in histogenetischer Beziehung zum Sympathicus, steht. Als die wichtigsten Etappen dieser Erkenntnis seien nur die folgenden angeführt. Stilling hat (1892) if die Chromreaktion an Zellen der Glandula intercarotica beobachtet, ferner bei Hund, Katze und Kaninchen (menschliches Material stand ihm in erforderlicher

1) Du Ganglion intercarotidien, l. c:; aus Prioritätsgründen ausführlich zitiert in "Die chromophilen Zellen und Körperchen des Sympathicus" desselben Autors, Anat. Anzeiger, 31. Dez, 1898, S. 229. 
Frische post mortem nicht zur Verfügung) analoge Zellgruppen in den Bauchganglien des Sympathicus, sowie aus solchen Zellen bestehende Körperchen, namentlich in der Nachbarschaft der Semilunarganglien. A. Kohn hat sich dann (1898 und die folgenden Jahre) unabhängig von Stillings Untersuchungen der Frage gewidmet und seinen Arbeiten ist die Auffassung der Gesamtheit der chromaffinen Zellen, Gewebe und Organe als eines Einheitlichen, eines Systems im Einzelindividuum wie in der Tierreihe za danken.

Zur Prüfung der Frage der Chromreaktion der Zellen der Coccygea wurden die Steissenden einer beträchtlichen Anzahl menschlicher Embryonen verschiedener Fötalperioden ${ }^{1}$ ), wie auch an zahlreichen Exemplaren die entsprechende Region des Neugeborenen an Serienschnitten und schliesslich auch neupräparierte Coccygeae des postuterinen Lebens verschiedenen Alters nach Fixation des Gewebes in der für die Chromreaktion von $\mathrm{Kohn}$ empfohlenen Weise untersucht.

Das Ergebnis war übereinstimmend und ausnahmslos ein negatives. Es erwiesen sich nicht nur die durch Form, Stellung und Protoplasmabeschaffenheit so charakteristischen Coccygeazellen selbst gegen die Chromierung refraktăr, sondern es ist mir auch niemals gelungen, zwischen diesen Zellen eingelagerte Einzelelemente mit positiver Chromreaktion zu sehen. ${ }^{2}$ )

Mit diesen wenigen Worten wăre das Ergebnis einer ziemlich langwierigen Untersuchungsreihe erledigt, ich kann mir aber nicht versagen, auch noch auf die dabei gewonnenen Befunde hinsichtlich der Beziehungen zwischen Steissdrüse und Sympathicus einzugehen, welche, wie mir scheint, in den bisherigen Darstellungen eine keineswegs einwandsfreie Schilderung erfahren und demgemäss zu Schlussfolgerungen geführt haben, welche nicht aufrecht $\mathrm{zu}$ erhalten sein dürften.

1) Für die gütige Unterstützung bei der Bearbeitung dieses Teiles meines Materiales bin ich Herrn Dr. H. Thaler, ehemaligem Hospitanten des Institutes, zu besonderem Dank verpflichtet

2) Bei Schaper, 1. c. (S. 214), beisst es: „Weder aus dem Text noch aus den der Walkerschen Arbuit beigegebenen Textfiguren ist etwas über das Vorhándensein gelb oder bräunlich gefärbter Zellen zu ersehen. Sollte dies vielleicht nur darauf zurückzuführen sein, dass Walker sich keiner Chromsalzlösungen zur Fixation seiner Organe bediente? ${ }^{2}$ Schaper hat die Tabelle anf Seite 156 der Walkerschen Arbeit übersehen, aus welcher in genauer Spezifizierung hervorgeht, dass in 28 Fällen im Gemisch von Müllerscher Flüssigkeit mit Formol fixiert worden war. 
Schon die grundlegende Beschreibung Luschkas ${ }^{1}$ ) bringt die bis zum heutigen Tage gültige diesbezügliche Anschaung in präziser Fassung (S. 55): "Ich habe dieses Organ, mit dem Vorbehalte, es für einen integrierenden Bestandteil des sympathischen Nervensystems zu erklären, einstweilen gewisser auusserer Qualitäten wegen in die sehr gemischte Gesellschaft der Drüsen ohne Ausführungsgang eingereiht."

Beim Studium der Begründung dieser Ansicht von seiten Luschkas tauchen unvermeidliche Zweifel auf. Es ist zu bewundern, welche feine Einzelheiten seinen anatomischen und mikroskopischen Untersuchungen zugänglich waren, aber die um ein halbes Jahrhundert weiterentwickelte mikroskopische Technik gestattet und $z$ wingt uns, eine Reihe seiner Angaben als unrichtige und irrtümliche zu bezeichnen. Wenn Luschka beispielsweise zwei Befunde yon Nervenfasern beschreibt, welche an der Oberfläche, resp. im Innern eines Steissdrüsenkörperchens mittels einer Ganglienzelle endigten, so kann ich mich eines Verdachtes nicht erwehren, der sich mir auch an einzelnen anderen Stellen seiner Schilderungen ergeben hat, dass er nämlich statt der Drüse, resp. statt einzelner Teildrüsen, gelegentlich beim Fötus und beim Neugeborenen verschiedenartige andere Gebilde dieser Gegend auspräpariert habe.

Ich möchte nur des genaueren auf Luschkas Mitteilungen über die Beziehung des Sympathicus zur Coccygea eingehen. $\mathrm{Er}$ beschreibt als erster die zarten Sympathicusästchen, „welche in der Mittellinie der unteren Steissbeinwirbel herablaufen und durch die rundliche Lücke in der Sehnenplatte des Afterbebers bindurchtreten. Es sind 2 bis 3 . Fadchen von ausserster Feinheit, welche die sehr verdünnte Fortsetzung des Stammes der Arteria sacralis media begleiten...." Es heisst dann weiter: „Nach dem Eintritt des Nerven in das Parenchym der Steissdrüse findet grösstenteils eine Auflösung desselben in Geflechte statt, welche mitunter eine wahrhaft netzförmige Anordnung $\mathrm{zu}$ erkennen geben." Dieser Eintritt „in das Parenchym" ist aber nur im Sinne der anatomischen Praparation. zu verstehen, nicht im mikroskopischen Sinne, denn es heisst dann gleich: „Mit nicht geringen Schwierigkeiten ist die Erforschung der Endigungsweise der Nervenfasern der Steissdrüse verknüpft. Meist entzieht

1) Der Hirnanhang und die Steissdrüse des Menschen. Berlin 1860. Archiv f. mikrosk. Anat. Bd. 69. 
sich dieselbe in der Faserhülle der Blasen und Schläuche dem Blicke gånzlich. Eine Art der Endigung habe ich jedoch im Parenchym jenes Organes wiederholt mit vollkommener Bestimmtheit erkannt . . . .," es folgt nun die Beschreibung der beiden erwähnten Fålle mit Endigung der Nervenfaser in eine Ganglienzelle. Es ist demnach Lu s chka ausser in diesen beiden Fallen nicht gelungen, die Nervenfasern weiter als bis zu den bindegewebigen Hüllen der Teildrüsen zu verfolgen und die erwăhnten "Geflechte" verlaufen in und mit diesen Hüllen. Das Gleiche gilt dann auch für. Luschkas bezügliche Angaben in der „Anatomie des menschlichen Beckens" (Tübingen 1864, S. 195).

In einer mit der eben vorgebrachten übereinstimmenden Auffassung führt auch Jacobsson ${ }^{1}$ ) den Befund Luschkas an, indem er von den zwei oder drei feinen Sympathicuszweigen spricht, welche „zur Drüse verlaufen, in deren Stroma sie späterhin eine plexusartige Verbreitung bilden. Ich kann die Richtigkeit dieser Angaben Luschkas . . . . bestätigen."

Ich habe nun an einer Reihe von lebenswarmen Früchten in späteren Abschnitten der Fötalzeit wie auch an Neugeborenen (unmittelbar post mortem) die Steissdrüse samt Umgebung in Serienschnitten (von 4 bis $6 \mu$ Schnittdicke) mittels des Ramon y Cajalschen Ammoniakalkohol-Verfahrens wie auch mittels der Markscheidenfärbung geprüft und kann diesbezüglich aussagen, dass in keinem der untersuchten Falle ein Eintreten von Nervenfasern, weder Remakscher noch markhaltiger in den Komplex, resp. die Komplexe der epithelahnlichen Coccygeazellen nachzuweisen war - wenn auch Nervenfaserbündel in nächster Nachbarschaft der Drüse, resp. der Teildrüsen, fast stets sichtbar waren. Es soll dieses Verhalten noch eingehender zur Sprache kommen, ich möchte zunächst aber versuchen, die Ursache dieses engen răumlichen Nebeneinander aus den Befunden der früheren Embryonalperioden zu erklären.

Beim Studium der Schnittserien vom Steissende junger Föten (von etwa $20 \mathrm{~mm}$ Scheitelsohlenlånge angefangen) gewann ich nămlich den Eindruck, dass die Entwicklung der Sympathicusramifikation derjenigen der $A$. sacr. media und insbesondere der Ausbildung der Ramifikation der letzteren voraneile, derart, dass

1) Beitr. zur Kenntnis der fötalen Entwicklung der Steissdrüse. Arch. f. mikr. Anat. u. Entwicklungsgesch., Bd. 53, 1899, S. 78 . 
sich die Arterie im Längswachstum ihres Endabschnittes, inbegriffen der Ästchen desselben, vielfach der präexistierenden Sympathicusramifikation gewissermassen als Geleise bedient. Eine strenge Gesetzmässigkeit der Verlaufsrichtung von Arterie und Nerv scheint dabei nicht zu bestehen; sowohl in der Sagittalwie in der Frontalebene tauschen sie gelegentlich ihren Platz. Erst in späteren Fötalperioden scheint sich diesbezüglich das typische Verbältnis herzustellen, jedoch gilt das nur für die Hauptståmmchen und nicht für die Ramifikation.

Auf diese Weise sieht man also zwar nicht allenthalben, aber überaus häufg, Sympathicusfaserbündel und Arterienstamm oder -ästchen im innigsten Kontakt, ja an manchen Stellen auch derart, dass jegliche Zwischengewebselemente zwischen ihnen $z u$ fehlen scheinen. Gerade gegen die Umbiegungsstelle um die Steissbeinspitze hin schien mir dieses Verhăltnis häufig besonders ausgeprägt - also an jener Stelle, in deren Bereiche die Steissdrüse hauptsăchlich zur Entwicklung kommt.

Die enge räumliche Beziehung zwischen A. sacr. media und $N$. sympathicus ist demnach von vornherein gegeben und besteht schon zu einer Zeit, wo von dem Vorhandensein einer Steissdrüse nichts erkennbar ist. $\mathrm{Zu}$ dieser Zeit ist das Verbalten des Zwischengewebes dieser Gegend ein gewissermassen noch indifferentes, erst spăter findet eine Strukturierung im Sinne der deutlichen und gesonderten Umhüllung einzelner Gebilde durch dichteres Zusammenrücken der Zwischengewebselemente an ihrer Peripherie statt, wie das zunăchst in der Umgebung der Kreuzund Steissbeinwirbel zu sehen ist. Aber auch beim Neugeborenen ist die Zwischengewebsgliederung bei weitem noch nicht in jener Schärfe zu sehen, welche sie in den Bildern des späteren Lebens zeigt.

Diese Beschaffenheit des Zwischengewebes scheint mir insbesondere bei den Verhältnissen jener bindegewebigen Anteile erwähnenswert, welche als Stroma und als Hülle oder Kapsel der Drüse, resp. der Teildrüsen beschrieben werden. Dem von Walker (l. c.) diesbezüglich Mitgeteilten, möchte ich noch folgendes anfügen. Beim Neugeborenen sieht man (mit Vorteil nach Färbung mittels der van Giesonschen Methode) wohl schon eine Art Kapsel um die. Drüse in Form einer ziemlich gleichmässig breiten Lage eines kernärmeren, gröberfaserigen Binde- 
gewebes; jedoch ist dasselbe gegen das benachbarte Bindegewebe an vielen Stellen unscharf abgesetzt, geht an solchen Stellen ohne Grenze in die Faserung der Nachbarschaft über und es umkreist nicht, wie in den späteren Lebensabschnitten, das Drüsenparenchym in ausgeprägten Zirkulärtouren. Auch das Drüsenstroma zeigt noch ein ganz anderes Aussehen, wie ja überhaupt der Bau der Drüse des Neugeborenen sich sehr wesentlich von dem der Drüse des späteren Lebens unterscheidet; die Drüse oder die Teildrüsen des Neugeborenen stellen je ein mehr kompaktes Ganzes mit einfacher Kontourierung in Form einer rundlichen oder ovalen, bisweilen auch einer hackenförmig um die Steissbeinspitze gekrümmten Bildung dar, innerhalb welcher allerdings die zukünftige konglomerierte Konfiguration schon angedeutet ist, indem die Drüsenzellen sich zu mantelartigen Lagen um je eines der kleinen Gefassslumina anordnen, deren stets eine gewisse Anzahl (etwa fünf bis zehn im Schnittbild einer mittelgrossen Einzeldrüse) in verschiedenartiger Verlautsrichtung in einer solchen Bildung zu sehen ist; zwischen diesen Mänteln sind dann auch mehrfach, aber nicht durchgreifend, sehr zarte und spïrliche Bindegewebslagen zu seben - die Anlage des späteren Drüsenstromas.

In der Regel ziehen nun - beim Neugeborenen - die Sympathicusfaserbündel, durch die besprochene Kapsel vom Drüsenparenchym getrennt, an der Drüse vorbei; gelegentlich findet sich aber auch ein (meist ganz dünnes) Bündel, welches in die Kapsel selbst eintritt und eventuell auch eine Strecke weit sich unmittelbar an die Peripherie des Parenchyms anlegen kann, um dann nach kurzem intrakapsulären Verlauf in unveränderter Stärke den Kapselbereich wieder zu verlassen. Es macht den Eindruck, als wäre das betreffende Nervenästchen bei Ausbildung der Kapsel mehr zufällig in dieselbe gefasst worden, hineingeraten.

Bei der postuterinen Entwicklung der Coccygea, welche wesentlich in einer Verlängerung jener Gefässchen, deren Lumina vorhin erwähnt wurden, samt umkleidenden Zellmänteln besteht (die Zahl der Zellagen um je ein Gefässlumen scheint mir nicht in gleichem Verhăltnis anzuwachsen, ${ }^{1}$ ) das. Protoplasma der

1) -Beim Nengeborenen und bei einem $34 \mathrm{~cm}$-Fötus.hatte ich Gclegeubeit, schöne Mitosen zu seheu, ich glaube aber, dass die Zellvermehrung hauptsächlich ein Längen- und weniger ein Dickenwachstum der Mäntel zur Folge hat. 
Einzelzelle nimmt aber deutlich an Volumen zu), wird der kompakte Verband der Einzeldrüse zur Formation eines etwa maulbeerartigen Gebildes gelockert. Bei diesem Auswachsen der von den Zellagen umscheideten Gefässe mag es wohl auch häufig zur Durch- und Umwachsung nachbarlich gelagerter Nervenabschnitte kommen. Auch die während der Wachstumsvergrösserung sicherlich häufig sich ergebende Verschmelzung benachbarter, ursprünglich gesonderter Teildrüsen $\mathrm{zu}$ gemeinsamen grösseren Gebilden mag das Bild einer Einverleibung von Nervenfaserbündeln in das Drüsenstroma gelegentlich hervorrufen. Dem früher beschriebenen, nämlich der innigen topischen Beziehung zwischen den Ramifikationen von Sympathicus und A. sacr. med. entsprechend, scheint mir die Annahme der Häufigkeit solcher "Pseudoeinverleibungs" - vorgange recht wahrscheinlich. Die Drüsen- „Kapsel " des Neugeborenen ist dabei wohl sicherlich kein endgültiges Gebilde und werden dem früher skizzierten weiteren Entwicklungsgang gemäss wohl mehrfach Anteile der ursprünglichen "Kapsel“ zu spăterem "Stroma"

Es erscheint mir nach dem Ausgeführten recht begreitlich, dass sich in der nächsten Umgebung der Drüse und mehrfach auch in den bindegewebigen Partien zwischen den Teildrüsen, resp. dann im "Stroma" der Drüse des späteren Lebens, Nervenfaserbündel in grösserer oder geringerer Reichlichleit nachweisen lassen, weil eben diese Bindegewebspartien vorwiegend Anteile des nervenreichen $Z$ wischengewebes der Coccygealgegend sind, welche erst allmählich im Laufe der Entwicklung und des Wachstums in das Gebiet der Drüse einbezogen wurden. Ich könnte auch durchaus nicht behaupten, dass der Reichtum an Nervenfasern des intraglandularen Zwischengewebes grösser sei als der des extraglandulären, vielmehr scheint mir eher das Gegenteil der Fall zu sein; die Art und Weise der Nervenverteilung im intraglandulären Gebiet scheint mir auch nicht die von seiten mehrerer Autoren gegebene Darstellung im Sinne einer „plexusartigen Anordnung" zu rechtfertigen.

Ich muss also auf Grund meiner histologischen Befunde eine innigere Beziehung (und zwar auch im Sinne der Histogenese) $z$ wischen Sympathicus und Steissdrüse entschieden in Abrede stellen und schliesse mich der Auffassung Arnold s ${ }^{1}$ ) an (natür-

1) Virch. Arch., Bd. 32, S. 293. 
lich nur im morphologischen und genetischen Sinne, mit Ausschaltung seiner irrtümlichen Deutung der Drüsenzellen), welche in seiner Namensgebung für die Drüse nglomeruli arteriosi coccygei" (1. c. S. 322) zum Ausdruck kommt; das formgebende und zunăchst in die Augen springende an der Steissdrüse sind die achsial in ihren Einzelanteilen verlaufenden Gefässchen, welche Arnold als zum Teil ampullenförmige Säcke, zuerst durch Injektion von der A. sacr. med. aus, als Ramifikationen derselben darzustellen in der Lage war. Diese typische achsiale Gefässlagerung entspricht auch den Verhältnissen bei den fötalen Bildern der Drüse, deren jüngstes mit Sicherheit agnoszierbares Stadium ich bei einem Fötus von $150 \mathrm{~mm}$ in Form einer Zellmasse gesehen $\mathrm{zu}$ haben glaube, welche in zwei- bis fünfacher Lage noch wenig protoplasmareicher, vorwiegend längsgestelltel ovaler Zellen die Arterie im Bereiche ibres Endabschnittes bis zur Steissbeinspitze und einzelne kleine Ästchen auf eine Strecke hin mantelartig umgab; eine morphologische scharfe Abgrenzung von den (in diesem Fötalstadium noch) plumpen Mediazellen war bier nicht allenthalben möglich, nur stellenweise war eine deutlichere Unterscheidung dadurch gesichert, dass die Drüsenzellen, wenn mit ausgesprochen längsovaler Form, der Gefassachse parallel, also mit ihrer Achse senkrecht auf die Langsachse der Mediazellen gelagert waren. ${ }^{1}$ ) Aber auch bei den spateren Stadien, in welchen die Drüse schon leicht erkennbar ist, indẹ ihre Form beilaufig der des Neugeborenen gleicht, kommt dieser achsiale Gefássverlauf, resp. die mantelartige Konfiguration der Drüsenzellmasse um das Endothelrohr unzweideutig zur Ansicht - ein Verhaltnis, welches, wie früher erwăhnt, dann auch, noch während des postuterinen Drüsenwachstums zu Recht besteht.

Auf die weiteren Einzelheiten der Darstellung Arnolds einzugehen, ist hier nicht der Platz. Dass er in den mịlkroskopischen Bildern von seinen Injektionsprăparaten die wirklichen

1) Tatsächlich glaube ich, veträchtlich jüngere Drüsenstadien in meinen Fötenserien geseben zu haben, nämlich in Niveau des Steissbeinendes gelegene Formationen unter dem Bilde einer uinschriebenen und ringsum gleichmässigen Mediazellwucherung an der Art. sacr, med., es wäre aber zur einwandsfreien Feststellung ibrer Steissdrüsennatur das verglejchende Studium der Bilder einer lückenlosen Reihe menschlicher Föten erforderlich gewesen, was mir mein Mlaterial leider nicht gestattete. 
Gefässendothelien im Drüsenbereiche nicht zu Gesicht bekam, sondern die Drüsenzellagen als einen lumenumkleidenden Anteil ansah, welcher in „unverhältnismässiger Entwicklung“ die Fortsetzung des Gefässendothels darstellen sollte, ist durch die Untersuchungen Sertolis ${ }^{1}$ ) und Eberths ${ }^{2}$ ) in endgültiger Weise als Irrtum gekennzeichnet und richtig gestellt worden. $\mathrm{Ob}$ die von Eberth für das Organ angewendete Benennung „Plexus vasculosus coccygeus" (S. 213) derjenigen Arnolds vorzuziehen sei, scheint mir eine nicht eben wesentliche Frage, ich akzeptiere lieber den Vorscblag Sertolis, vorläufig "die alte, vom Entdecker ihm gegebene Benennung "Steissdrüse " beizubehalten, ", in der Erwartung, dass neue Untersuchungen etwas Licht über die Funktion dieses Organs bringen werden." Beiläufig gesagt, hat sich letztere Erwartung bisher noch nicht erfüllt und wird, wie ich befürchte, noch lange unerfüllt bleiben. Dafür scheint mir der Umstand zu sprechen, dass trotz sehr zahlreicher Exstirpationen der Drüse bisher noch niemals von spezifischen Ausfallserscheinungen berichtet worden ist; ich meine nămlich die so häufigen Fälle von Resektion des Kreuz- und Steissbeines, welche ja gegenwärtig in ausgedehnter Weise von den Chirurgen vorgenommen werden und zweifellos eine radikale Entfernung der Drüse mit sich bringen; obwohl eine grosse Zabl solcher Fălle viele Jahre hindurch, beispielsweise wegen der Gefahr eines Karkinomrezidivs, genau in Evidenz gehalten werden, ist noch niemals über Symptome, welche im Sinne von Ausfallserscheinungen gedeutet werden könnten, berichtet worden. Vielleicht wird doch noch der vergleichenden Anatomie, trotz der diesbezüglich wenig befriedigenden bisherigen Ergebnisse, in der Coccygeafrage die Aufklärung vorbehalten bleiben.

Ich hatte im früheren Gelegenheit gehabt, meine Zweifel über die Befunde Luschkas hinsichtlich der innigeren Beziehung zwischen Sympathicus und Drüse $\mathrm{zu}$ begründen. Ähñlicher Zweifel ergibt sich mir bei der einzigen modernen Publikation, welche sich. vorwiegend entwicklungsgeschichtlich, mit diesem Teil der Coccygeafrage, und zwar gleichsinnig mit Luschkas Ergebnissen, beschaftigt, der bereits zitierten Veröffentlichung Jacobssons.

1) Virch. Arch., Bd. 42, 1868, S. 370.

2) Strickers Handbuch der Lehre von den Gew., 1. Bd., 1871, S. 209. 
An einem Fötus von $11 \mathrm{~cm}$ Körperlänge findet er $150 \mu$ über der Steissbeinspitze ein zellreiches Gebilde, Arterie, Vene und sympathische Nerven enthaltend: „Die Nerven, von denen die Gefässe dicht umsponnen werden, enthalten ausser Fasern noch zahlreiche Zellen; hauptsäenlich die letzteren verleihen der betreffenden Partie ihr kompaktes Aussehen." Weiter kranialwärts 500 (!) $\mu$ von der Steissbeinspitze ein analoges Knötchen. „Beide Zellenanhäufungen dürften . . . a auf die Drüsenanlage Bezug haben." Bei einem $24 \mathrm{~cm}$ langen Fötus werden dann, unter Hinweis auf diese beiden Knötchen, ebenfalls zwei analoge Bildungen, 60 und $430 \mu$ über der Steissbeinspitze, besprochen und werden diese Bildangen als Steissdrüsen en miniature" bezeichnet.

Zum erstenmal begegnet er .der Steissdrüse als einem selbständigen und scharf markierten Gebilde" bei einem $15 \mathrm{~cm}$ langen Fötus. Sie lagert in der Gabel zwischen $A$. sacr. med. und einem Ästchen der Steissbeinspitzenumbiegung, ersterer entsprechend, und ist bereits bindegewebig abgekapselt. „Der dicht vorbeiziehende Sympathicus gibt an die Drüse mehrere Äste ab, die in mebreren Verästelungen durch die Kapsel zwischen die Parenchymzellen der Drüse hinein" dringen, "wo man sie dann als feine von Eosin schwach rot gefärbte Fibrillen verfolgen kann. In welchem Verbältnis diese Nervenfasern zu den Parenchymzellen stehen, erhellt nicht aus den Bildern." Die Drüse erscheine nur spärlich und zwar in ihren Randpartien vaskularisiert.

Ich stehe nicht an, bezüglich aller dieser Gebilde, deren Beschreibung nach Jacobssons Darstellung hier in Kürze wiedergegeben wurde, mit Entschiedenheit anzunehmen, dass sie mit der Steissdrüse durchaus nichts zu tun haben. Er selbst charakterisiert die Gebilde am besten, wenn er bezüglich des zuletzterwähnten sagt, es stehe „dieses Zellklümpchen in einer so engen Verbindung mit dem sympathischen Ganglienstrange and entspricht auch in seiner Lage dessen unterem Ende, dass man gezwungenerweise das Parenchym als einen abgeschnürten Teil desselben, und die Zellen als modifizierte Ganglienzellen betrachten muss."

Jacobsson hat die "sympathischen Bildungszellen" und ihre weiteren Entwicklungsstadien, mit deren Bildern wir heute - dank der Forschungen der eingangs erwähnten Autoren vertraut sind, nicht gekannt, sonst hätte er die bisher angeführten Gebilde richtig klassifiziert, nämlich als junge chromaffine Körperchen.

Seine Föten bis zur Körperlange yon $24 \mathrm{~cm}$ sind in Sublimat, Formalin oder Alkohol tixiert, es bestand also keine Möglichkeit der Chromreaktion, nur der $11 \mathrm{~cm}$ und der $12,2 \mathrm{~cm}$ lange in Müllerscher Flüssigkeit; aber auch bei denen von $24, \check{\partial} \mathrm{cm}$ bis 
zur Reife, welche in letzterer Flüssigkeit fixiert waren, scheinen die chromaffinen Elemente seiner Beachtung entgangen zu sein.

$\mathrm{Zu}$ dieser Auffassung zwingen mich verschiedene Umstände; zunächst die Topographie und die Beschreibung der kleineren Gebilde, insbesondere dann aber auch die Abbildung und Schilderung der grösseren Bildung vom $15 \mathrm{~cm}$ langen Fötus.

Ausschlaggebend gerade bezüglich der letzteren erscheinen mir zwei Momente in der Darstellung Jacobssons: seine Angaben über das Verbalten der Gefässe und diejenigen über den Nervenbefund. An Gefässen, heisst es in der Beschreibung des grösseren Gebildes vom $15 \mathrm{~cm}$ langen Fötus, ,gibt es . . . ăusserst wenige; nur sehr spärliche Kapillaren kommen in der Kapsel und den am meisten peripherisch gelegenen Teilen des Parenchyms vor." Dem früher Ausgeführten gemăss, scheint mir dieser an Schnittserien erhobene Befund allein schon für die Annahme massgebend, dass das beschriebene Gebilde mit der Steissdrüse nicht identisch ist - eben wegen des Feblens eines zentralen arteriellen Gefässchens. Die (zitierte) Beschreibung der in das Parenchym eintretenden, als eosinrote Fibrillen zwischen die Parenchymzellen verfolgbaren sympathischen Nervenfasern, entspricht genau demjenigen, was man immer wieder, insbesondere auch an den jungen Formen der chromaffinen Körperchen, sehen kann und steht im diametralen Gegensatz zu allen Bildern, welche ich von der fötalen Steissdrüse gewonnen habe.

Bezüglich der Drüse des nächstgrösseren Fötus $(24 \mathrm{~cm}$ Körperlänge) wird ausdrücklich auf ihr differentes Aussehen hingewiesen: „Anstatt der äusserst spärlichen Kapillaren, welche wir in der Kapsel und den peripherischen Teilen der Drüse fanden, sehen wir hier die ganze Drüsenmasse von einer Menge sinuös erweiterter vorkapillarer Arterien durchzogen." Die zugehörige Abbildung (Fig. 7) bringt dieses Verhalten auch klar zum Ausdruck. Hinsichtlich der Nerven beschränkt sich die Beschreibung leider auf den Satz: „Nerven ziehen von dem dicht nebenan gelegenen Sympathicus reichlich in das Organ ein " und die Abbildung lässt sie vollkommen vermissen. Wären sie in diesem Falle mit gleicher Eindeutigkeit zu sehen gewesen, wie im vorangehenden, so wäre vielleicht eine eingehendere $\mathrm{Be}$ schreibung zu erwarten gewesen, und ich wage den $Z$ weifel auszusprechen, ob dasjenige, was er in diesem Falle an seinen 
(15 $\mu$ dicken) Serienschnitten als Nervenfasern gedeutet hat, nicht vielmehr Anteile des $Z$ wischengewebes waren, von welchem es heisst: "Von der Kapsel schieben sich kleinere Bindegewebsstreifen in das Parenchym hinein."

In der Beschreibung der weiteren Stadien, bis zum Erwachsenen, kommt das Verhalten der Nerven nicht mehr zur Sprache. Auch in der Zusammenfassung wird auf den intraglandulären Nervenbefund nur anlässlich der Erwähnung des Befundes beim $15 \mathrm{~cm}$ Fötus hingewiesen; im weiteren heisst es dann: „Dagegen müssen wir die Entstehung der Drüse aus dem Sympathicus als mehr denn wahrscheinlich erachten. Die primäre Drüsenanlage erscheint auf dem Platze des kaudalen Endes der Sympathicusanlage und hängt bereits von ihrem ersten Auftreten an und fortwährend mit dem Sympathicus nahe zusammen."

Den Beweis für die Richtigkeit letzterer Annahme einer genetischen Beziehung zwischen Drüse und Sympathicus scheint mir Jacobsson nicht erbracht $z u$ haben und meine eigenen Befunde sprechen durchaus dagegen; ich muss auch seiner Darstellung: „Die Gefässe spielen . bei der Entstehung des Organs eine sekundäre Rolle und wachsen erst, nachdem dieses bereits als ein selbständiger, distinkt markierter und gefăssloser Zellenhaufen angelegt worden, in dasselbe hinein" mit Rücksicht darauf entgegentreten, dass sich die Anschauung Jacobssons ausschliesslich auf die Befunde gerade jener im früheren genauer besprochenen Gebilde zu stützen scheint, welche ich nicht als Frühstadien der Steissdrüsenentwicklung gelten lassen kann, sondern als chromaffine Körperchen ansprechen muss. Vielmehr habe ich aus meinen Fötenserien die Anschauung gewonnen, dass die ersten Formationen der Drüse an die Art. sacr. media resp. ihre Ästchen gebunden sind, welche sie als eine mantelartig angeordnete Zellmasse konzentrisch umlagern.

Die Darstellung Jacobssons über die Entstehung der Steissdrüse hat zur Folge gehabt, dass dieselbe als ein Glied in die Reihe der chromaffinen Gebilde reklamiert wurde, insbesondere seitdem das Gleiche für die Carotisdrüse, welche ja in manchen Punkten Ähnlichkeit mit der Steissdrüse aufweist, mit unzweideutigem Ergebnis ausgeführt worden war. So schreibt $\operatorname{Kohn}^{1}$ ) (S. 311): ${ }_{n}$ Seit ihrer Entdeckung durch L uschka wird die Steiss- 
drüse immer in einem Atem mit der Carotisdrüse genannt. Von allen Wandlungen der Ansichten sind beide gemeinsam betroffen worden. Auch diesmal dürfte die Steissdrüse diesem Schicksale nicht entgeben. Die Untersuchungen Jacobssons haben ihre Abkunft rom Sympathicus sehr wahrscheinlich gemacht. Damit wäre eine wesentliche Vorbedingung für ihre Aufnabme unter die Paraganglien erfüllt." "Carotis- und Steissdrüse sind aber nur die zwei Endglieder der fortlaufenden Paraganglienkette, die dem Sympathicus angeschlossen ist."

Minder vorsichtig und umfänglicher hat Schaper (wie einleitend besprochen wurde) eine gleichsinnige Hypothese im darauffolgenden Jahre vertreten.

Es wurde im bisherigen - dem Vorschlag Sertolis entsprechend - von der Steissdrüse und von Drüsenzellen gesprochen. Damit sollte aber natürlich bezüglich der tatsächlichen Natur der Coccygea nicht präjudiziert werden. Die Frage, ob dem Protoplasma der Zellen der Steissdrüse eine spezifische (physikalische oder chemische) Funktion zukommt, entzieht sich vorlăufig der Beantwortung, wenn auch manches morphologische Detail für eine solche Annabme $\mathrm{zu}$ sprechen scheint; abgesehen von der überaus reichlichen Vaskularisation ${ }^{1}$ ) würde der Annahme vielleicht auch die eigentümliche Protoplasmabeschaffenheit der Drüsenzellen nicht widersprechen. Es lässt sich nămlich an den embryonalen Drüsenbildern verschiedener Altersstufen verfolgen, wie die ursprünglich spindelige Zellform mehr und mebr in die ovale und gegen das Ende der Fötalzeit in eine unregelmässig rundliche (resp. infolge gegenseitiger Abplattung der Zellen polygonale) übergeht, proportionell der zunehmenden Succulenz des Protoplasmas, welche vom Ende der Fötalzeit an bleibend in der für die Steissdrüsenzelle so charakteristischen Protoplasmaunfärbbarkeit zum Ausdruck kommt: es macht den Eindruck, als würde der Kern inmitten einer die ganze Zelle erfüllenden Vakuole schweben. Nur an einzelnen Zellen wird gelegentlich ein zart farbbarer peripherer Protoplasmaanteil sichtbar. Ich habe alle in Betracht kommenden Färbungsmethoden (an Schnitten gehärteter Objekte, Gefrier-

1) Vergl. hierzu Walker (l. c.) 
schnitte kamen nicht in Anwendung) mit negativem Erfolg angewendet, ebenso die Reaktionen auf Glykogen und Fett. Insbesondere sei auch das negative Ergebnis der Schleimreaktionen betont.

Fielleicht steht zu dem eben besprochenen auch die überaus hăufig zur Beobachtung kommende eigentümliche Beschaffenheit des an die Drüsenzellen zunächst angrenzenden $Z$ wischengewebes in Beziehung: es zeigt nämlich — von den spăteren Stadien des Fötallebens an - eine Anordnung in auffallend weiten, oft fast rundlichen Maschen, wobei der Mascheninhalt gleichfalls bei den verschiedenartigsten Tinktionsmethoden ungefärbt bleibt.

Die Verfolgung des Entwicklungsganges der Steissdrüse lehrt, dass sie mit keinem der in Betracht kommenden Nachbar-

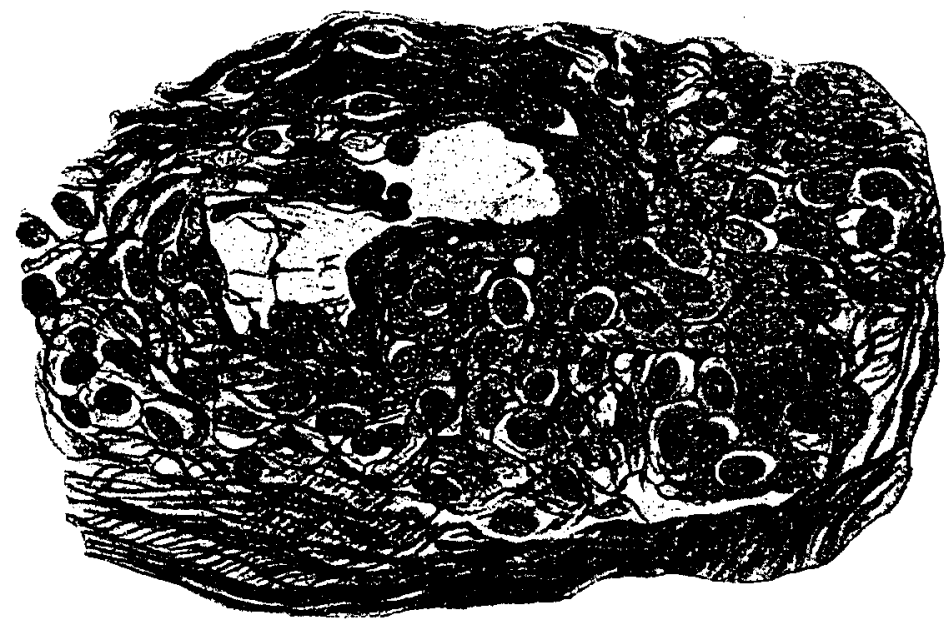

Fig. 1.

Spezif. Bindegewebsfärbuug. Vergr. 600/1.

organe (Canalis neurentericus, kaudales Chordaende, Steisswirbel; vergl. hierzu auch Ja c obs s o n, l. c., S. 95) in genetische Beziehung gesetzt werden kann, ${ }^{1}$ ) und nachdem ich mich bemüht habe, ein

1) Andere nur gelegentlich in nachbarliche Boziehung zar Drüse tretende Gebilde bedürfen hierza keiner Berücksichtigung; ich meine damit zunächst die bisweilen in auffallender Reichlichkeit vorhandenen Gebilde nach $A r t$ der $P$ a cin ischen Körperchen, insbesondere aber auch jene anormulen Formationen, welchen in der Histogenese der Sakralgeschwälste eine wichtige Rolle zukommt, über die in jüngster Zeit H. A. Thaler (Deut. Zeitschr. f. Chir., Bd. 79, S. 112) und R. Me je r (Virch. Arch., Bd. 180, S. 334) berichtel haben. 
Gleiches für den Sympathicus darzutun, käme gewissermassen schon per exclusionem nur mehr die Art. sacr. media in Betracht. Wesentlicher als diese Exclusion ist aber der Befund der Bilder früher Fötalperioden, in welchen die Steissdrüse, wie erwähnt, als umschriebene kurzspindelförmige Wandverdickung der Art. sacr. med. in Form einer ringsum gleichmässigen, die Gefăssperipherie bildenden kleinen Zellmasse erscheint; deren spindelige Elemente zunächst von den Mediazellen nicht unterscheidbar sind. ") Die morphologische Übereinstimmung mit den kontraktilen Mediazellen, welche einen nicht unbeträchtlichen Abschnitt des

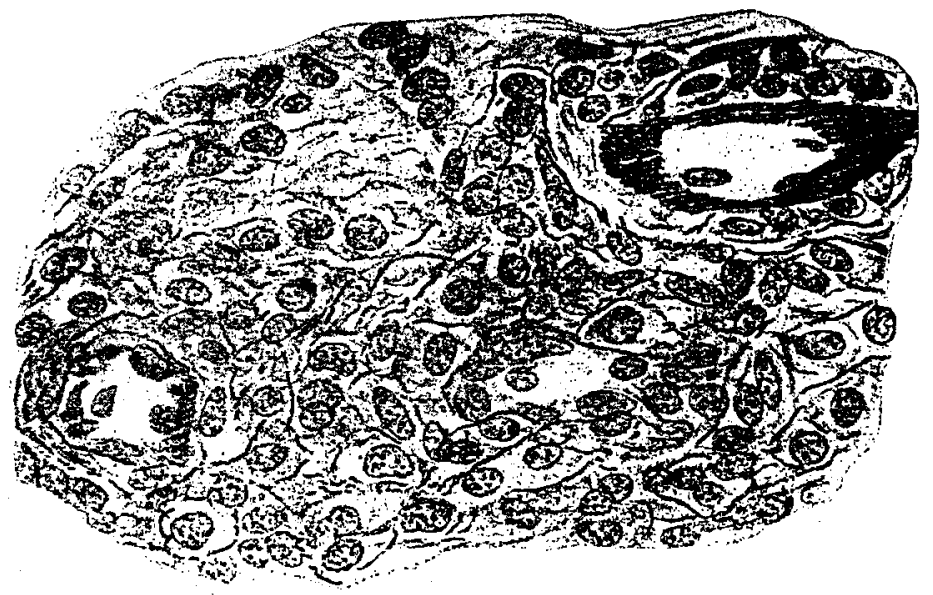

Fig. 2

Spezif. Elasticafärbung. Vergr. 600/1.

Fötallebens hindurch besteht, ist dabei vielleicht doch mehr als eine zufälligè Ähnlichkeit răumlich aneinander grenzender Gebilde.

Vielleicht ist eine andere Beobachtung gleichsinnig verwertbar. Es lässt sich wiederholt konstatieren, dass eine in den Drüsenbereich eintretende Arterie ihre Media verliert, also mit kapillarer Wandbeschaffenheit ihren Weg fortsetzt - als wären die Mediazellen durch die Drüsenzellen abgelöst worden.

Ein weiteres Moment, welches ich bier heranziehen möchte, ist der Befund, welcher sich (bei Anwendung spezifischer Färbungen)

1) Bemerkenswert erscheint auch die gelegentliche Multiplizität solcher Bildungen in ganz gleichartiger Form, auch in relativ grösseren Abständen -voneinander, beispielsweise vor und hinter dem Steissbein gelagert. 
sowohl für Bindegewebs- wie auch für elastische Fasern im Bereiche der Drüsenzellen ergibt. Bezüglich beider Faserarten zeigt sich nämlich eine von der Wand des Zentralgefässes des betreffenden Drüsenanteiles ausstrahlende Ausbreitung zwischen die Drüsenzellen, in nächster Nachbarschaft des Zentralgefässes letztere stellenweise geradezu umspinnend, peripherwărts sich allmählich verlierend. ${ }^{1}$ ) An der Drüsen- resp. Teildrüsenperipherie zeigt sich häufig ein ăhnliches Eindringen beider Faserarten und zwar in entgegengesetzter Richtung, zentripetal, meist nur auf die kurze Erstreckung von 2 bis 3 Zellagen, vom umgebenden Stroma her. (Bezüglich letzteren Befundes liesse sich an die Möglichkeit eines peripheren, gleichsam „infiltrativen“ Drüsenwachstums denken; gegen eine soiche Erklärung müsste ich einwenden, dass ich Mitosen niemals in dieser Randschichte, sondern immer nur inmitten der Zellmantelbreite gesehen habe.)

Die Pindegewebslagen um das zarte achsiale Gefass sind oft recht reichlich, die elastischen Fasern 'spärlich, resp. letztere bestehen meist nur aus einer einzigen, dem Endothel anliegenden Faserlage, welche also einer Elastica interna entsprechen würde. Die von diesen Faserlagen abzweigenden, intercellulär sich verästelnden Bindegewebs- und elastischen Fasern scheinen mir in denjenigen Drüsenanteilen, welche das achsiale Gefäss nahe seinem Eintritt umgeben, am reichlichsten zu sein.

Dieser Befund einer intimen Beziehung der beiden Faserarten zu den Drüsenzellen scheint mir mit einer Vorstellung der letzteren als metamorphosierten Mediaelementen in einem gewissen Einklang zu stehen.

Natürlich batte ein solcher Interpretationsyersuch nur den Wert einer - noch sehr stützbedürftigen - Hypothese.

Es wăre vielleicht gerade im Anschluss an die letzter Ausführungen verlockend, auf die von einer Reihe von Autoren vertretene Anschauung der „perithelialen“ Natur der Steissdrüse einzugehen. Ich möchte mich aber dessen vor allem aus dem Grunde enthalten, weil die Fassung des Begriffes des „perithelialen “ nach den bisherigen Darstellungen durchaus keine einheitliche

1) Vergl. hierzn auch die übereinstimmende Angabe von HlebKoszanska ("Peritheliom der Lu s chk a schen Steissdrüse im Kindesalter") Zieglers Beiträge, Bd. 35, 1904, S. 589. 
ist und diesbezügliche Erörterungen den Rahmen des Vorliegenden weitaus überschreiten müssten. Ich beabsichtige mich an anderer Stelle mit dieser Angelegenheit zu beschäftigen.

\section{Resumé.}

1. Die Zellen der Steissdrüse geben weder im fötalen noch im postfötalen Leben die Chromreaktion.

2. Eine histogenetische Beziehung zum Sympathicus ist nicht vorhanden.

3. Vielmehr ist eine solche Beziehung $z u$ den Mediaelementen der Arteria sacralis media, respektive ihrer $\ddot{A} s t c h e n$, mit einiger Wahrscheinlichkeit zu vermuten.

Nachschrift. Nach Abschluss dieses Manuskriptes erlangte ich noch Kenntnis der Publikation v. Schumachers \#Über die Nerven des Schwanzes der Säugetiere und des Menschen, mit besonderer Berücksichtigung des sympathischen Grenzstranges ${ }^{2},{ }^{1}$ ) in welcher (pag. 601) über sein negatives Ergebnis bei Untersuchung der Chromreaktion der Zellen zweier lebenswarm fixierter Steissdrüsen berichtet wird. In mündlicher Besprechung teilte mir der Autor mit, dass er, wie eine in Bälde abzuschliessende Untersuchungsreihe zeigen wird, bezüglich der Histogenese der Steissdrüse zu Ergebnissen kommt, welche mit denjenigen des Vorliegenden durchaus übereinstimmen.

2) Sitzungsber. d. K. Akad. d. Wissensch. in Wien, matl.-naturw. Kl., 1905 